\title{
Osteoglycin - linking bone and energy homeostasis
}

osteoglycin
in humans is
linked with
regulating
glucose
metabolism
during
changes
in energy
homeostasis,
which supports
the findings
in mice

Bone mass adjusts to increases in body weight through mechanical stimuli; however, whether bone can regulate the energy required for this process is unknown. Now, new research indicates that osteoglycin, a proteoglycan also known as mimecan, can regulate bone formation to match changes in whole body energy homeostasis in mice. The study by Herbert Herzog and colleagues also shows that in humans, osteoglycin is associated with changes in BMI, weight and glucose levels.

"This work began following our study that demonstrated that neuropeptide Y (NPY) pathways from the brain exert powerful control over the skeleton," explains Herzog. The study showed that NPY signalling regulated bone mass, insulin secretion and glucose homeostasis in mice, which prompted the researchers to search for the mediator responsible for communicating between the bone and pancreas.

In the present study, microarray and mass spectrometry analyses of osteoblasts from NPY1 receptor-deficient mice were used to identify osteoglycin as a novel factor linking bone to insulin regulation. To understand the role of osteoglycin in the body, the researchers used

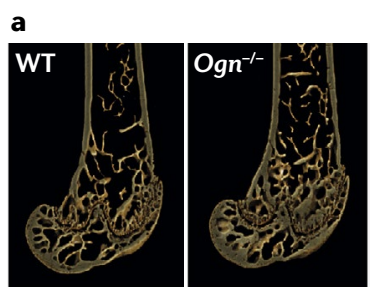

b

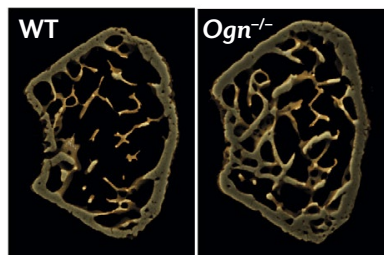

Representative sagittal (part a) and cross-sectional (part $\mathbf{b}$ ) images from femora of $\mathrm{Ogn}^{-/-}$and wild-type (WT) mice. Adapted with permission from Lee, N. J. et al. Mol. Metab. https://doi.org/10.1016/j.molmet.2018.05.004 (2018), Elsevier.
CRISPR-Cas9 technology to knock out osteoglycin expression in mice $\left(O g n^{-/-}\right)$. Osteoglycin-deficient mice fed a high-fat diet (HFD) or normal chow for 5 weeks exhibited substantial impairment of glucose tolerance as well as insulin resistance, compared with wild-type mice fed with the same diets. Conversely, injection of osteoglycin into wild-type mice fed with normal chow resulted in improved whole body glucose metabolism compared with mice treated with buffer. The researchers demonstrated that this effect is due to osteoglycin-enhancing insulin action.

Next, Herzog and co-workers investigated the role of osteoglycin in bone homeostasis. $\mathrm{Ogn}^{-1-}$ mice showed increased BMD, bone mineral content and trabecular bone volume compared with wild-type mice. This increase in bone mass was associated with increased osteoblast activity and demonstrates that osteoglycin regulates bone formation.

To understand the link between bone formation and energy metabolism, the researchers induced obesity in wild-type mice through a HFD. The authors reported that obese mice had decreased circulating levels of osteoglcyin compared with control mice fed normal chow. This effect was negatively associated with bone mass, which suggests that osteoglycin promotes alterations in bone mass following changes in body weight during intervals of high-fat feeding.

Subsequently, the researchers fed wild-type and $\mathrm{Ogn}^{-/-}$mice a HFD and, as expected, both groups exhibited increased bone volume compared with mice fed normal chow. Interestingly, only the obese wild-type mice showed increased trabecular bone volume, and not $O g n^{-/-}$mice fed a HFD. This effect suggests that osteoglycin is a novel mediator in regulating bone mass, particularly in trabecular bone, in response to changing energy homeostasis during increase body weight.

Finally, Herzog and co-workers examined the role of osteoglycin in humans during changes in energy homeostasis by examining the weight and plasma levels of osteoglycin and glucose in 22 patients with severe obesity who underwent weight loss by either a dietary programme, gastric sleeve or gastric bypass surgery. Before the weight loss intervention, there was a negative correlation between osteoglycin levels and BMI, and a positive correlation between osteoglycin levels and lean mass. After the intervention, patients who had undergone substantial weight loss had a considerable increase in serum levels of osteoglycin, which correlated with reduced blood levels of glucose. Therefore, osteoglycin in humans is linked with regulating glucose metabolism during changes in energy homeostasis, which supports the findings in mice.

"Osteoglycin functions to regulate insulin responsiveness in accordance with energy balance to aide skeletal adaptation to energy and weight change," concludes Herzog.

Ivone Leong

ORIGINAL ARTICLE Lee, N. J. et al. Osteoglycin, a novel coordinator of bone and glucose homeostasis. Mol. Metab. https://doi.org/ 10.1016/j.molmet.2018.05.004 (2018) 